ful, but resulted in extensive ecchymosis. Perhaps the most certain method of arresting such a haemorrhage is that adopted by the writer, namely, the passing of a suture round the cyst itself ; certainly no ill effect was observed from this.

Finally, it is interesting to speculate as to how far the presence of pertussis was a contributory factor.

REFERENCES

1. Rowland, W. P.-Jl. Ophthal. Otol. and Laryng., Vol. XXXIII, pp. 177-180, 1929.

2. Briggs, A. H.-Personal communication.

\title{
A CASE OF ACUTE UNILATERAL RETRO-BULBAR NEURITIS ASSOCIATED WITH NASAL-SINUS DISEASE
}

BY

R. R. James, Sir StClair Thomson, Lionel Colledge and H. Graham Hodgson, C.V.O.

LONDON

\section{Introduction. (R.R.J.)}

RETRO-BULBAR neuritis, due to nasal sinusitis, is probably much less common than was formerly thought. It is true that a retrobulbar neuritis may occasionally be due to absorption from some septic focus. I have seen such a case in a patient with severe dental sepsis. The eye.condition ran an acute course, was bilateral and cleared up completely in about four months' time.

In my early days at St. George's Hospital I saw a chronic type of case. Mr. Harold Barwell found evidence of nasal sinus trouble; the man was operated on and went out with much improved vision. A few years later the condition recurred and the patient was sent to Mr. J. B. Lawford who wrote to me for the n'tes of the case. In writing to thank me for them Mr. Lawford told me that, on reviewing the case, he had come to the conclusion that the ocular condition was entirely due to tobacco!

The case which we now report would seem to be of some rarity.

The Nasal History. (SIR STC. T.)

The patient first came under observation in 1930 with evidence of suppuration in the left maxillary sinus. He was then 72 years of age and, being averse from a larger operation, the sinus was simply drained through an opening in an empty molar socket. 


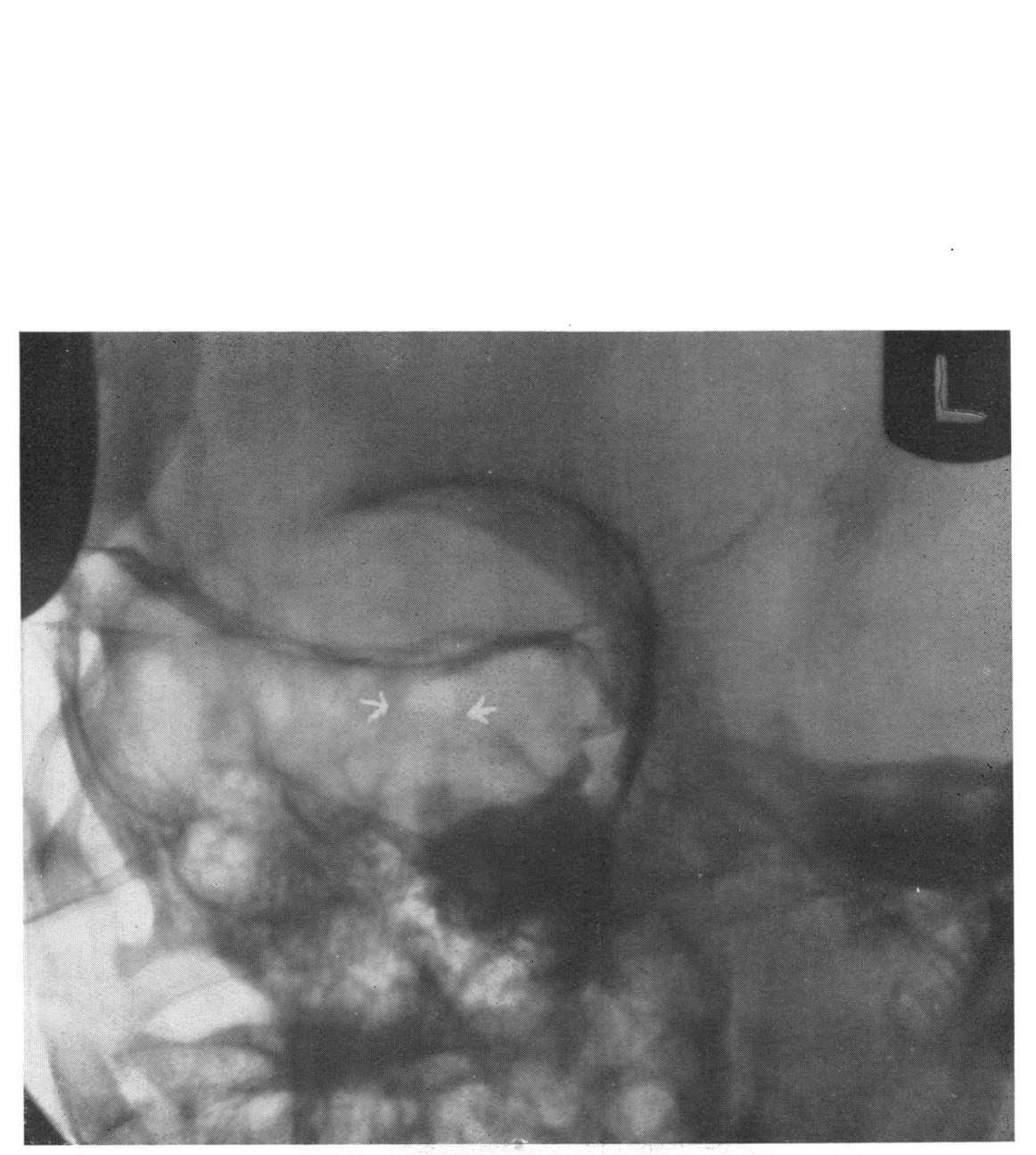

The Left Obligue View.

The posterior ethmoidal cells are blurred and ill defined as the result of infection. One of these cells is indicated by arrows and contains a small amount of fluid. 


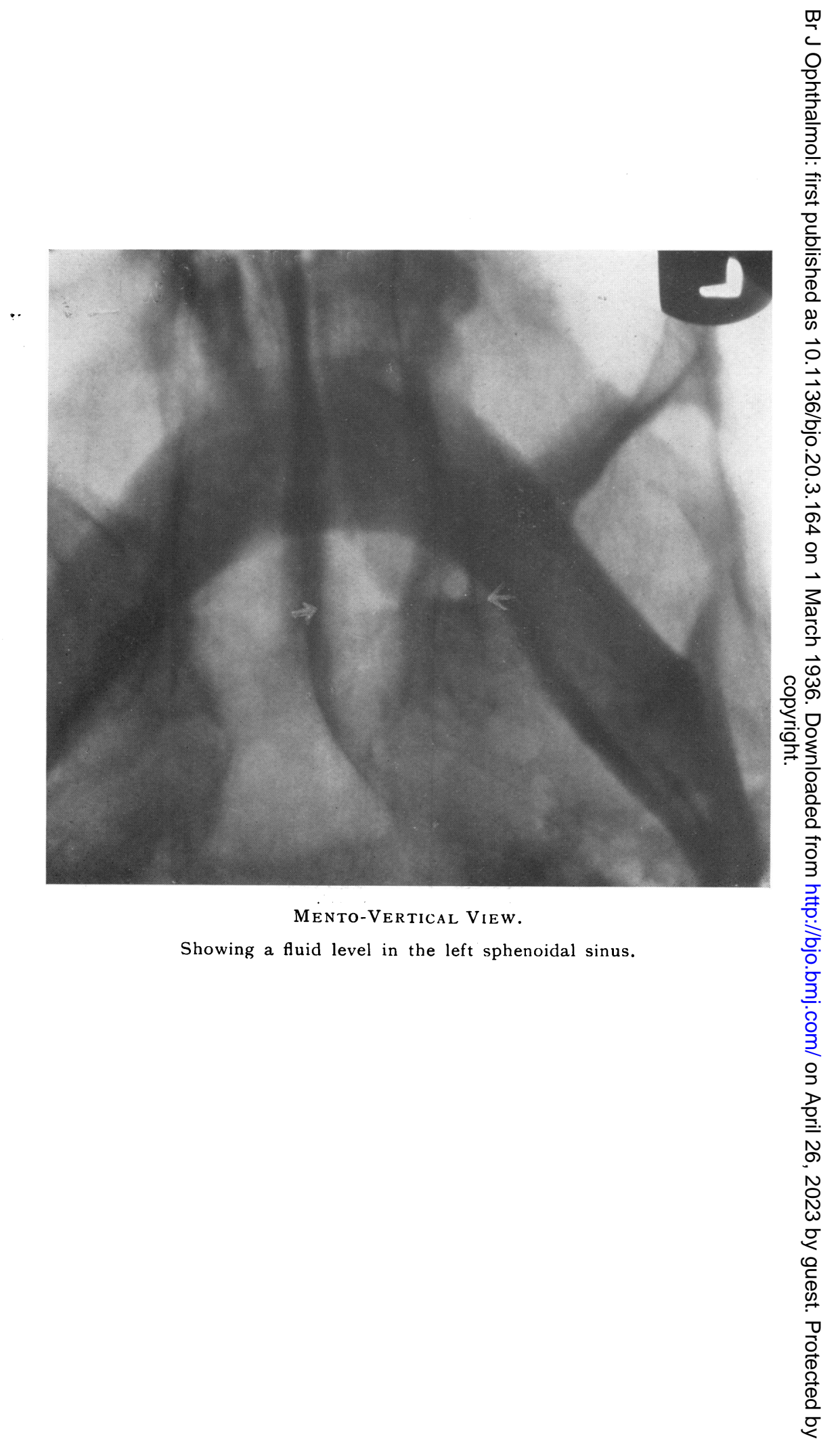


This gave so much relief that he was not seen for two years. In May, 1932, he returned to say that the rubber obturator in this molar socket had been worn too long and that, the flange having perished, it had slipped into the cavity of the antrum! An $\mathrm{X}$-ray plate demonstrated this and also showed some thickening of the mucosa and some cloudiness of the left frontal sinus. The other sinuses were then clear. It was therefore decided to operate on the maxillary sinus and an endo-nasal operation was performed on May 2, 1932.

All symptoms disappeared except an occasional discharge of muco-pus from the region of the left frontal sinus and the patient was not seen from December, 1932 until he was referred to me on May 10,1933 , by Mr. R. R. James with the symptoms elsewhere described.

\section{The Ophthalmological History. (R.R.J.)}

A medical man, aged 75 years, who had previously attended for change of glasses and mild attacks of conjunctivitis since 1923, called on May 8, 1933.

Up to this date the vision, after correction of a small amount of compound hypermetropic astigmatism, had been $6 / 6$ and Jaeger 1 . in each eye.

The recent history was that he had woken up on May $\tau$ to find the sight of the left eye blurred. There was also some pain on movement of the globe.

The right eye was normal in every respect, with $6 / 6$ vision after correction.

The pupil reaction to light on the left side was extremely sluggish ; tension was normal ; vision, only hand movements.

After dilating the pupil the following note was made. "Lens and vitreous clear, disc flat. No haemorrhage present." The field was full save for a slight contraction, in the upper nasal quadrant, and there was a large central scotoma.

The blood pressure was reported as $200 / 110$.

There was a history of previous antral trouble on the left side, for which Sir StClair Thomson had performed a radical operation a few years before, with excellent results. Vide supra.

Sir StClair saw the patient and reported a clear left frontal sinus; the left antrum was washed out and only a tiny spot of mucus resulted.

Dr. Graham Hodgson saw the patient on May 11 and his report was in our hands early on May 12. The following is a copy.

"All radiograms were taken in the erect position.

There is thickening of the lining membrane in the left antrum and in the left frontal sinus and left ethmoid cells and also to a 
slight extent in the right frontal sinus and right anterior ethmoid cells. The right antrum is radiologically normal.

The posterior ethmoid cells on both sides show thickening of the lining membrane, which is more marked on the left side than on the right. At the time of the examination some fluid, the level indicated by arrows, was present in one of the left posterior ethmoid cells.

Both sphenoidal sinuses show thickening of the lining membrane, this being more marked in the left sphenoidal sinus than in the right, and in the left sphenoidal sinus at the time of the examination, a fluid level, also indicated by arrows, was present."

The deterioration of vision was very rapid. On the morning of May 12 the eye was practically blind. The pupil hardly acted to light, only bare perception of light was present and no field could be charted. The left disc was swollen $1.0 \mathrm{D}$. and the vessels were slightly turgid.

Sir StClair Thomson strongly advised immediate operation and that afternoon $\mathrm{Mr}$. Colledge opened and drained the left sphenoidal and ethmoidal cells.

The following are his remarks :-

This patient was sent to me by Sir StClair Thomson with the history so carefully detailed by $\mathrm{Mr}$. James. Under cocaine and adrenalin anaesthesia there was visible a pulsating spot of light on the purulent discharge in the neighbourhood of the ostium of the sphenoidal sinus, resembling the pulsating spot often seen in the neighbourhood of a perforation in the tympanic membrane when drainage is insufficient. With Hajek's forceps the anterior wall of the sphenoidal sinus was cut away and some posterior ethmoidal cells opened. Complete healing took place and all discharge from the nose ceased after a few applications of argyrol. A large opening remains into the sphenoidal sinus.

Unfortunately, although the operation was done within five days of the time when symptoms first declared themselves, no improvement in vision resulted. The damage was apparently due to a direct spread through the wall of the sinus into the optic nerve.

Although this route of infection is well-known, it seems actually to be rare, and the great majority of cases of retro-bulbar neuritis, referred for examination, prove to be early cases of disseminated sclerosis or, sometimes, tobacco amblyopia. In a few cases infection of the tonsils or accessory sinuses acts as an area of focal sepsis. Direct extension as in this case is rare and it no doubt accounted for the symptom of pain on movement of the eye.

Another interesting feature of the case was the clear demonstration by Dr. Graham Hodgson of a fluid level in the sphenoidal sinus. 
In spite of treatment no improvement in vision occurred. On May 16 the left pupil was inactive to light and by June 28 the fundus examination showed a secondary type of optic atrophy with some narrowing of the vessels. 'The right eye was normal throughout and, when seen in November 1935, was quite healthy, with $6 / 6$ vision after correction.

We infer that the massive lesion of the optic nerve was due to a direct spread of infection from a focus in the posterior ethmoidal cells and sphenoidal sinus. If so it must have occurred quite at the apex of the orbit. It will be noted that about a couple of months elapsed before the signs of a descending atrophy of the nerve were established by fundus examination. This length of time agrees pretty closely with what one expects in cases of direct tearing across of the nerve in cases of fractured base of the skull.

Perhaps after all our patient was fortunate in that the infection confined itself entirely to the nerve and that no backward spread into the cavernous sinus took place. The line that divides two such possibilities must be an exceedingly fine one.

\section{THE EXTRACTION OF CATARACT BY THE ELECTRO-DIAFACO METHOD \\ (after Lopez Lacarrère of Madrid)}

BY

\section{KhaliL}

CAIRO

Cataract is an eye disease which necessitates the removal of the affected lens. Of all the methods used from the time of Daviel, 1745 , till the present day, that which resulted in the extraction of the greater part of the diseased lens was considered best. Much better still are those methods which end in the removal of the lens in its capsule. Of the various intra-capsular methods known up to the present, one may mention (1) the category of expression -as Smith's method; (2) the category of suction-as by Barraquer's method; (3) the category of catching the lens with a blunt forceps, dislocating it, and extracting it out of the eye-as done by Elschnig, Kalt, Stanculeanu, Sinclair and others ; (4) the category of removal of lens after electro-coagulation-as originated by Jess and Lopez Lacarrère. It is out of the question that I would make a comparison between this last method and the various methods of intra-capsular extraction of cataract mentioned. However, it is as always useful to increase one's armamentarium in combating diseases. My object is to describe and demonstrate by pictures 